\title{
Actualización: La atención de pacientes lesbianas, gays y bisexuales y transgénero (primera parte)
}

The care of lesbian, gay, bisexual and transgender patients (first part)

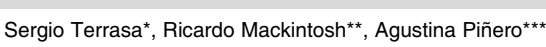

\begin{abstract}
Resumen
Los autores de este artículo describen y discuten: 1) una conceptualización de las diferentes orientaciones sexuales, y de las dificultades para acordar definiciones y para determinar la prevalencia de las mismas; 2) la problemática del estrés de las minorías y su asociación a resultados adversos de la salud mental; 3) las barreras reportadas de acceso a los servicios de salud y algunas estrategias para atenuarlas, especialmente las que buscan facilitar la comunicación; 4) algunos aspectos particulares de control periódico de salud en esta población, haciendo especial hincapié en el rastreo de cáncer de cérvix en las mujeres que tienen sexo con mujeres y en el de cáncer anal en la población que practica el sexo anal receptivo; 5) los principales resultados de estudios observacionales que evaluaron el desarrollo de niños criados en familias homoparentales. Una segunda parte de este artículo se explayará en la los cuidados de salud de los pacientes con disforia de género.
\end{abstract}

\section{Abstract}

The authors of this paper describe and discuss: 1) a conceptualization of the different sexual orientations, as well as the difficulty in agreeing about definitions and to determine their prevalence; 2) the issue of minority stress and its association with adverse mental health outcome; 3) reported barriers to health services access and some strategies to mitigate them, especially those proposed to facilitate communication; 4) some particular aspects of the periodic health control of this population, with particular emphasis on cervical cancer screening in women who have sex with women and anal cancer in people who practice receptive anal sex; 5) the main results from observational studies that evaluated the development of children raised in homparental families.

A second part of this article will discuss about the health care of patients with gender dysphoria.

Palabras clave: lesbiana, gay, bisexual, homosexual, cuidados preventivos, barreras a los servicios de salud, estrategias de comunicación. Key words: lesbian, gay, bisexual, homosexual, preventive care, barriers to health services, comunicational strategies.

Terrasa S, Mackintosh R y Piñero A. La atención de pacientes lesbianas, gays y bisexuales y transgénero (primera entrega). Evid Act Pract Ambul. 14(2).57-62. Abr Jun. 2011

\section{El problema de las definiciones y de la determinación de la prevalencia de las diferentes orientaciones sexuales}

Se define orientación sexual a la atracción constante hacia otra persona en el plano emotivo, romántico, sexual o afectivo, clasificando algunos expertos a la orientación sexual de acuerdo a tres grandes áreas: 1) la de los deseos; 2) la de las conductas; 3) la de la identidad de género ${ }^{\dagger+}$.

Por ejemplo, para definirla, Klein y col. ${ }^{1}$ tienen en cuenta varios aspectos: la atracción sexual, la conducta sexual, las fantasías sexuales, las preferencias emocionales, las preferencias sociales, la autoidentificación y el estilo de vida.

Estas diferentes dimensiones de la orientación sexual hacen particularmente difícil definir la prevalencia de "homosexualidad" o "bisexualidad". Como mínimo ejemplo de esta dificultad, reproducimos algunos resultados de la histórica investigación de Kinsey y col. ${ }^{2}$, cuyos principales hallazgos "taxonómicos" se resumen en la tabla 1; ó los del trabajo de Laumann y col. $^{3}$ resumidos en la tabla 2 .

Tabla 1: principales resultados sobre el deseo, la reactividad y la conducta sexual reportados por Kinsey y col.

\begin{tabular}{|c|c|c|c|c|}
\hline & & & \multicolumn{2}{|c|}{ Frecuencia } \\
\hline & & & Varones & Mujeres \\
\hline \multicolumn{3}{|c|}{ Excitación y/o masturbación fantaseando con el sexo opuesto } & 84 a $89 \%$ & 64 a $69 \%$ \\
\hline \multirow[t]{2}{*}{ Respuesta o experiencia bisexual } & \multicolumn{2}{|c|}{ Actividad sexual o "reactividad" hacia ambos sexos } & $46 \%$ & \\
\hline & \multicolumn{2}{|c|}{ Reactividad equivalente hacia ambos sexos } & $11 \%$ & 4 a $7 \%$ \\
\hline \multirow[t]{5}{*}{ Respuesta o experiencia homosexual } & \multicolumn{2}{|c|}{ Al menos un orgasmo relacionado con una persona del mismo sexo } & $37 \%$ & $13 \%$ \\
\hline & \multirow[t]{2}{*}{ Entre los 16 y los 55 años } & Predominantemente homosexual & $10 \%$ & \\
\hline & & Exclusivamente homosexual & $8 \%$ & \\
\hline & \multirow[t]{2}{*}{ Entre los 20 y los 35 años } & \multicolumn{2}{|l|}{ Casi exclusivamente homosexual } & 2 a $6 \%$ \\
\hline & & \multicolumn{2}{|l|}{ No casadas exclusivamente homosexuales } & 1 a $3 \%$ \\
\hline
\end{tabular}

Resumido de: Kinsey A y col. (1948/1998) Sexual Behavior in the Human Male; (1953/1998) Sexual Behavior in the Human Female; y Gebhard P y col. (1979/1998) The Kinsey Data: Marginal Tabulations of 1938-1963. Interviews Conducted by the Institute for Sex Research. Philadelphia: W.B. Saunders; Bloomington, IN: Indiana U. Press. Versión resumida disponible en URL: http://www.iub.edu/ kinsey/resources/ak-data.html\#Findings

\footnotetext{
"La identidad de género es el sentimiento psicológico de ser hombre o mujer teniendo en cuenta el papel social que conllevan los géneros, básicamente la adhesión a ciertas normas culturales relacionadas con el comportamiento femenino o masculino.

* Servicio de Medicina Familiar y Comunitaria de Hospital Italiano de Buenos Aires. Departamento de Salud Pública del Instituto Universitario Hospital Italiano de Buenos Aires. Federación Argentina de Medicina Familiar y General (sede Metropolitana: Asociación Argentina de Medicina Familiar) sergio.terrasa@ hospitalitaliano.org.ar

** Servicio de Clínica Médica del Centro de Investigaciones Médicas e Investigación Clínica Norberto Quirno (CEMIC). mack44@fibertel.com.ar

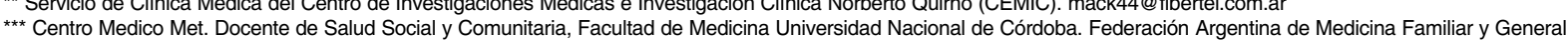
Asociación Cordobesa de Medicina Familiar. agusep@ hotmail.com
} 
Tabla 2: prevalencia de homo o bisexualidad en Estados Unidos (año 2000).

\begin{tabular}{l|c|c|c}
\multicolumn{2}{c|}{} & $\begin{array}{c}\text { Poblaciòn } \\
\text { general }\end{array}$ & $\begin{array}{c}\text { Grandes centros } \\
\text { urbanos }\end{array}$ \\
\hline \multirow{2}{*}{ Mujeres } & Identidad & $1,4 \%$ & $2,6 \%$ \\
\cline { 2 - 4 } & Conducta & $4,3 \%$ & $4,6 \%$ \\
\hline \multirow{2}{*}{ Hombres } & Identidad & $2,9 \%$ & $9,2 \%$ \\
\cline { 2 - 4 } & Conducta & $9,1 \%$ & $15,8 \%$ \\
\hline
\end{tabular}

Fuente: Laumann O y col. The Social Organization of Sexuality: Sexual Practices in the United States. Chicago: University of Chicago Press, 1994. Citado por: Dean L, et al. Lesbian, Gay, Bisexual, and Transgender Health: Findings and Concerns. Journal of the Gay and Lesbian Medical Association, Vol. 4, No. 3, 2000.

Por ejemplo y luego de tener en cuenta la dificultad mencionada previamente para estimar la prevalencia de las orientaciones sexuales minoritarias, se estima que entre 5 y $10 \%$ de la población podría ser calificada de lesbiana, gay u homosexual. En este sentido, podemos denominar lesbiana o gay a alguien cuyas relaciones emocionales y/o sexuales (y su proyecto de vida) son principalmente con alguien del mismo sexo. Sin embargo, si bien un $3 \%$ de los varones que atendemos ha tenido sexo varones, muchos de ellos se consideran heterosexuales ${ }^{4}$. O bien, muchas mujeres que se consideran "lesbianas" viven en celibato o bien son sexualmente activas con alguien del mismo o del otro sexo.

Dado que, teóricamente sólo podríamos determinar prevalencia la de atracción sexual y conductas, consideramos que no conviene hablar del "colectivo" de personas homosexuales, ya que englobaría a poblaciones diferentes; pudiendo ser razonable referirse a al colectivo de "personas que tienen una orientación sexual minoritaria” (OSM).
El estrés de las minorías y los problemas de salud mental derivados del mismo

El estudio de la salud mental de las poblaciones con OSM, ha estado teñido de una gran discusión respecto de si la homosexualidad debía ser o no ser considerada una enfermedad mental, especialmente durante la los años sesenta e inicios de los setenta de siglo pasado. Si bien este debate concluyó en 1973, año en que la homosexualidad fue eliminada de las categorías de la segunda edición del Manual Diagnóstico y Estadístico de los Trastornos Mentales de la Asociación Norteamericana de Psiquiatría (DSM II) ${ }^{5}$, ha continuado la discusión sobre si las minorías sexuales sufren una mayor carga de problemas de salud mental, derivados de la estigmatización a la que suelen estar sometidos.

Según Pearling , estrés es: "...toda condición que activa la maquinaria adaptativa del individuo...". Cuando hablamos de personas con una OSM, el estigma, el prejuicio y la segregación provocan un ambiente social estresante ${ }^{7}$. Este estrés se relaciona con las experiencias de eventos objetivamente hostiles, con las expectativas de rechazo, con la reacción defensiva de "ocultamiento" de la OSM y con el consiguiente deterioro de las capacidades de afrontamiento (en inglés "coping"). Por ejemplo, algunos autores hablan del concepto de homofobia internalizada y en el DSM IV $^{8}$ se hace referencia a que la homosexualidad puede cursar en forma egodistónica o egosintónica.

Este estrés crónico estaría implicado en el desarrollo y el mantenimiento de algunos problemas de salud mental cuya prevalencia es mayor en la población con OSM y que han sido documentado en numerosos trabajos, como el meta-análisis de King y col. ${ }^{9}$, cuyos resultados se resumen en la tabla 3 . Si bien sus resultados pueden estar sujetos a sesgos de selección, p. ej. que los individuos que integraron la muestra no hayan sido representativos de la población general- y de clasificación, $p$. ej. algunos definieron la orientación sexual basándose solo en la conducta reportada durante el último año- constituyen la mejor evidencia con la que contamos al día de la fecha sobre esta asociación.

Tabla 3: principales resultados de la revisión sistemática de King y col. sobre la asociación entre pertenecer a una minoría sexual y sufrir algún trastorno de la esfera mental.

\begin{tabular}{|c|c|c|c|c|}
\hline & Incidencia & $\begin{array}{c}\text { LGB } \\
\text { n/total }\end{array}$ & $\begin{array}{l}\text { No LGB } \\
\text { n/total }\end{array}$ & RR (IC95\%) \\
\hline \multirow[t]{2}{*}{ A lo largo de la vida } & Intento de suicidio (12*: 61,3\%) & $564 / 4845$ & $1652 / 33243$ & $2,47(1,87$ a 3,28$)$ \\
\hline & Depresión $\left(1^{2 *}: 0 \%\right)$ & $63 / 153$ & $1294 / 6852$ & $2,03(1,7$ a 2,41$)$ \\
\hline \multirow[t]{3}{*}{ A lo largo de 12 meses } & Ansiedad $\left(1^{2 *}: 0 \%\right)$ & $66 / 518$ & $1836 / 23215$ & $1,54(1,23$ a 1,92$)$ \\
\hline & Dependencia al alcohol $(12 *: 0 \%)$ & $76 / 753$ & $1271 / 27436$ & $2,2(1,78$ a 2,77$)$ \\
\hline & Dependencia a otras drogas (12*: $0 \%)$ & $26 / 518$ & $423 / 23215$ & $2,73(1,86$ a 4,02$)$ \\
\hline
\end{tabular}

LGB: población lesbiana, gay o bisexual. Fuente: King M y col. A systematic review of mental disorder, suicide, and deliberate self harm in lesbian, gay and bisexual people. BMC Psychiatry. doi:10.1186/1471-244X-8-70. http://www.biomedcentral.com/1471-244X/8/70

El estrés de las minorías se suma ${ }^{\ddagger} a$ las demás situaciones estresantes que tienen los individuos que no pertenecen a ellas, es crónico y de base social.

Podría definirse al estrés de la minoría como el resultado de la activación crónica de los mecanismos adaptativos -con el gasto de energía crónico que esto implica- en forma consecutiva a eventos hostiles "objetivos" del ambiente y a una decodificación "subjetiva" que hace el individuo perteneciente a la minoría de algunas señales del entorno, que pueden no ser realmente hostiles pero son vivenciadas como tales debido a su experiencia previa con situaciones que sí lo fueron. Ver el modelo teórico propuesto por Meyer en la figura 1.
Figura 1: modelo teórico propuesto por Meyer para conceptualizar la problemática del estrés de las minorías sexuales.

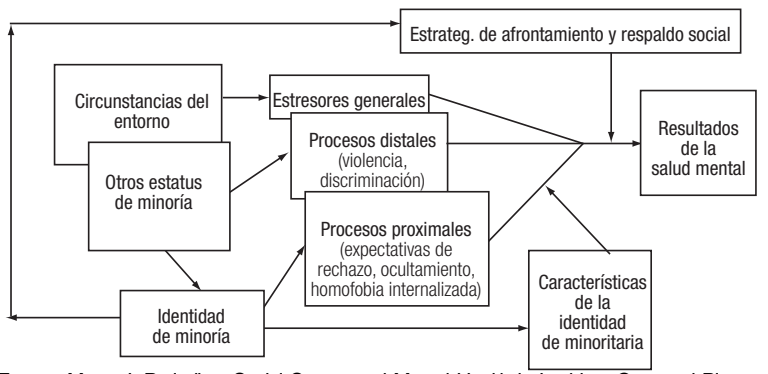

Fuente: Meyer I. Prejudice, Social Stress, and Mental Health in Lesbian, Gay, and Bisexua Populations: Conceptual Issues and Research Evidence. Psychol Bull. 2003 September 129(5): 674-697.

\#Además, a diferencia de otras minorías, pueden sufrir la "doble segregación": la de la comunidad y la de sus propias familias de origen. 


\section{Las barreras al acceso de los Servicios de Salud}

Existe evidencia de que muchas personas con OSM demoran su atención o reciben cuidados inapropiados por su percepción de homofobia en el sistema de salud, que como dijimos puede ser real o no. Los datos de un trabajo realizado con datos poblacionales de 2003 a 2005 del Sistema de Salud Canadiense ${ }^{10}$ informan que, comparada con la población heterosexual, la población con OSM tiene menor probabilidad (aproximadamente $50 \%$ ) de reconocer a un profesional que les brinde cuidados longitudinales, mayor percepción de insatisfac- ción respecto de haber encontrado respuesta a sus problemas de salud (aproximadamente el doble) y menor tasa de realización de rastreo de cáncer de cérvix en los últimos tres años (64\% en la población lesbiana vs. $77 \%$ en las mujeres heterosexuales).

La tabla 4 esquematiza una adaptación propia del modelo de Knight ${ }^{4}$ que intenta explicar los obstáculos que tienen las personas pertenecientes a una minoría sexual para acceder a los cuidados de la salud, dividiéndolos en tres categorías: 1) del paciente; 2) del profesional; y 3) del sistema de salud.

Tabla 4: adaptación del modelo de Knight para explicar los obstáculos que tienen las minorías sexuales para acceder a los cuidados de la salud.

\begin{tabular}{l|l|c}
\multicolumn{1}{c|}{ Del paciente } & \multicolumn{1}{c|}{ Del profesional } & Del sistema \\
\hline $\begin{array}{l}\text { Vergüenza, ocultamiento y temor a la reacción del } \\
\text { profesional. }\end{array}$ & $\begin{array}{l}\text { Incomodidad, falta de entrenamiento en como preguntar, } \\
\text { eventual heterosexismo }{ }^{\prime \#}, \text { homofobia u hostilidad. }\end{array}$ & Entorno poco amigable. \\
\hline $\begin{array}{l}\text { Desconocimiento de algunos riesgos y de algunas } \\
\text { herramientas preventivas. }\end{array}$ & $\begin{array}{l}\text { Desconocimiento de conductas de su paciente, } \\
\text { desinformación sobre herramientas preventivas. Falta de } \\
\text { tiempo, temor a abrir la "caja de Pandora". }\end{array}$ & $\begin{array}{l}\text { Falta de guías de cuidados preventivos } \\
\text { basadas en la evidencia. Algunas } \\
\text { prácticas preventivas pueden no estar } \\
\text { cubiertas. }\end{array}$ \\
\hline $\begin{array}{l}\text { Temor a perder la cobertura, a que se entere el } \\
\text { empleador, etc. }\end{array}$ & Mal manejo de la confidencialidad. & Posible discriminación de personas de \\
\hline
\end{tabular}

Fuente: Adaptado de Knight D. Health Care Screening for Men Who Have Sex with MenAm Fam Physician 2004;69:2149-56.

Muchos expertos de países anglosajones sostienen que las personas con orientación sexual minoritaria "escanean" visualmente las instalaciones donde son atendidos buscando pistas que los ayuden a determinar si van a sentirse cómodos compartiendo información con los profesionales con quienes se van a relacionar. Por eso y para hacer amigable el entorno, algunos consensos recomiendan que se coloque algún cartel en el que se diga que la calidad de la atención que se brinda en esa institución no está condicionada por factores como edad, raza, religión, capacidades físicas o mentales, religión, orientación y/o identidad sexual; o bien algún ícono representativo que comunique este tipo de mensaje como los que se muestran en la figura $2 \mathrm{a}$, recordando que la exclusiva presencia de otros que no comunican este tipo de mensaje (ver figura $2 b$ ) puede contribuir a la sensación de segregación.

Figura 2: íconos que, de estar en la sala de espera, comunican que la calidad de la atención que se brinda en esa institución no está condicionada por la orientación y/o identidad sexual (2a) y que no lo comunican o pueden sugerir el mensaje contrario si aparecen en forma aislada $(2 b)$

2a

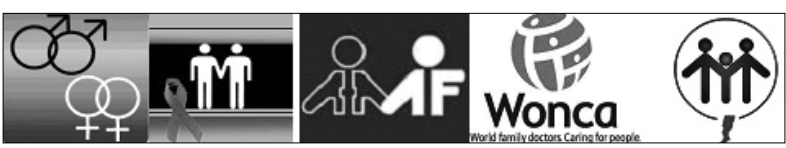

$2 b$
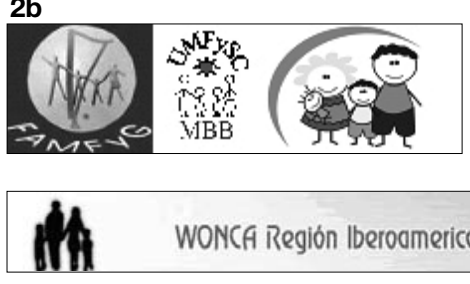

WONCA Región Iberoamericona CIMF
Nuestra experiencia en la práctica clínica nos dice que lo que más observan son las actitudes del entrevistador, el tipo de preguntas que éste formula y sus respuestas verbales y no verbales consecutivas a la información que éste va recabado a lo largo de la entrevista.

Algunos profesionales y estudiantes de carreras vinculadas con la Salud consideran que no habría que indagar sobre la orientación sexual de las personas y que sólo bastaría con preguntar acerca de conductas objetivas de riesgo (sexualidad no monogámica, sexo no protegido, etc.) con el fin exclusivo de recomendar las prácticas preventivas pertinentes (rastreo de enfermedades de transmisión sexual, vacunación anti hepatitis $A$ y $B$ ) ya que saber sobre los deseos y sobre la identidad sexual no modificaría la conducta clínica posterior.

Sin embargo, existe evidencia bibliográfica ${ }^{10}$ (y nuestra experiencia clínica lo confirma) de que la mayoría de las personas con OSM (las encuestas revelan un $90 \%$ ) desean poder compartir su orientación sexual con el profesional de la salud que les brinda cuidados médicos. Sostienen que esta posibilidad de conversar los hace sentir más cómodos en la entrevista, aumenta su percepción de que se les está brindando un cuidado integral, de que el profesional de la salud los considera una persona, que sienten que para ser mejor cuidados el equipo tratante debe conocer cuestiones de su vida personal.

Lo cierto es que sólo entre el $9 \%^{12}$ y el $30 \%$ de los profesionales le pregunta a sus pacientes sobre su orientación sexual y de los que lo hacen, sólo el $13 \%$ usa preguntas abiertas ${ }^{13}$. Probablemente, esto conduzca a que 50 a $80 \%$ de los pacientes con OSM refieran no revelar su orientación sexual. Si bien hemos podido identificar pocas publicaciones sobre las barreras en la comunicación de las poblaciones con OSM, el $30 \%$ refirío percibir una reacción negativa en el profesional de la salud cuando le comunicó su orientación sexual ${ }^{10}$ y sólo el $20 \%$ de los MSM refieren haber hablado abiertamente sobre ETS con sus médicos ${ }^{4}$

\#+Heterosexismo es un concepto que se refiere a la creencia de que la heterosexualidad es una forma de vida más saludable que la homosexualidad o la bisexualidad. Si bien no tiene connotaciones despectivas ni segregatorias, podría contribuir a las barreras de la atención de la salud por parte del profesional o del sistema de salud. 


\section{Estrategias para facilitar la comunicación}

Para facilitar la comunicación entre el profesional de la salud y el usuario del sistema recomendamos utilizar preguntas lo más abiertas posibles, no "juzgadoras" y, eventualmente, con introducciones que permitan al paciente contestar con la mayor libertad posible $e^{14,15}$. Una alternativa, es comenzar diciendo que uno pregunta estas cosas rutinariamente y/o preguntarlas durante el curso del resto del interrogatorio. Algunos ejemplos pueden ser los siguientes:

En el contexto de su control de salud, yo tengo que conocer cierta información, de la que a veces es incómodo hablar. Por ejemplo, ¿ha tenido sexo con personas de su mismo sexo?

A la hora de interrogar sobre el estado relacional de nuestro paciente, recomendamos comenzar usando palabras sin "género" como pareja, pareja sexual, persona, conviviente, amante, unión civil; o bien preguntando:

¿Con quién vive?, ¿Está usted en pareja?, ¿Hay alguna persona significativa en su vida?, ¿Cómo se llama su pareja?

Aunque no lo comente abiertamente, mucha gente ha tenido relaciones con personas del mismo sexo.

¿Las ha tenido usted?

Otra estrategia facilitadora que varios de nuestros colegas nos han comunicado es la realización del genograma, preguntando durante su confección, el nombre de pila la pareja, conviviente, o persona significativa para el paciente.

Otras formas de preguntar serían:

¿Tiene usted actividad sexual?, ¿Cuándo fue la última vez?, ¿Ha tenido sexo con hombres, con mujeres o con ambos?, ¿Cuántas parejas ha tenido?, ¿Ha usado preservativo?, ¿Qué tipo de relación a tenido (genital, anal, bucal, etc.)? ¿Mantiene usted una pareja "abierta" o una pareja "cerrada"?

Teniendo en cuenta que, como el título de este material lo expresa, no entendamos el significado de algo que nos está contando, puede ser necesario pedirle al paciente que nos explique con sus palabras la prácticas sexuales a las que se refiere; o bien ofrecerle que no nos conteste si por el momento se siente incómodo, difiriendo la pregunta hasta otro encuentro y "negociando" lo que quede escrito en la historia clínica.

Aunque pueda resultar obvia esta advertencia, recomendamos evitar términos vulgares para referirse a tal o cual orientación sexual. Si bien, es posible que los mismos pacientes los utilicen con sentido del humor, para evitar herir susceptibilidades es necesario tener una gran confianza con ellos y usarlas sólo en el momento justo. Por eso, una forma de evitar meternos en problemas es utilizando palabras que se refieran a la conducta -básicamente, el blanco de nuestras intervenciones preventivas- y no a la orientación. Como dijimos, hay muchos varones que tienen sexo anal con varones y que se definen a si mismos como heterosexuales. Entonces ante una situación como la descripta priorizaremos el conocimiento que tiene ese individuo sobre la implicancia de su conducta y sobre las prácticas de sexo seguro.

Siguiendo con esta línea de pensamiento, recomendamos a los profesionales de la salud que sean muy cuidadosos en el diseño y/o la elección de los cuestionarios estructurados que suelen utilizarse para "ganar tiempo" antes de la consulta con el médico, generalmente en la primera visita de un control de salud. Estos cuestionarios, por ejemplo suelen incluir preguntas, muchas veces con opción cerrada de respuestas como las siguientes: 1) Sexo (femenino o masculino); 2) estado civil (soltero, casado, divorciado o viudo).

Para resumir, recomendamos hacer foco en la conducta por sobre la identidad sexual; que las preguntas sean abiertas, sin especificidad de género y manteniéndonos atentos a que la sexualidad es dinámica y puede ser cambiante.

Recordamos que la sexualidad es un continuo (no suele haber "blancos" y "negros") y que se trata de un tema difícil de ser conversado. Por ejemplo, una persona puede tener una pareja estable pero "abierta".

Las dificultades están en los pacientes y en el proveedor de salud. En este sentido, la longitudinalidad en los cuidados suele ser de ayuda.

\section{El control periódico de salud de las personas con orienta- ciones sexuales minoritarias}

El primer punto que queremos destacar es que el control de salud de estas poblaciones es similar al de cualquier persona de su sexo y edad. Sin embargo y dada la mayor probabilidad que estas poblaciones se vean afectadas por problemas de salud mental, varios consensos de expertos ${ }^{8},{ }_{16}$ recomiendan realizar rastreo de trastornos de ansiedad, del ánimo y de la alimentación y de violencia doméstica (recomendaciones tipo $\mathrm{C}$ derivadas de consensos de expertos ${ }^{9}$ ).

Otro punto en el que hacen especial hincapié estos consensos es el de la toma de la historia sexual ${ }^{17}$, el rastreo de conductas de riesgo y la consejería para la prevención de enfermedades transmisión sexual ${ }^{18}$ (incluyendo el uso de métodos de barrera para las prácticas sexuales manuales o con juguetes sexuales) y el de inmunización contra hepatitis $A$ y $\mathrm{B}^{13}$ (todas recomendaciones tipo $\mathrm{C}$ ). Nuestra postura al respecto es que esta conducta debería derivar de la evaluación del riesgo de cada individuo (conducta) más allá de su orientación sexual.

Un comentario especial lo merecen el rastreo de cáncer de cérvix y el rastreo de cáncer anal.

\section{Rastreo de cáncer del cuello uterino}

Respecto del cáncer de cérvix, existe amplio consenso que la población lesbiana debe ser sometida a los mismos controles preventivos que la población general. Esta recomendación, ampliamente consensuada se basa en que el virus del papiloma humano (HPV) también se transmite por relaciones sexuales entre mujeres ${ }^{19}$, especialmente si comparten juguetes sexuales, y en que las mujeres que en el momento de la consulta sólo tienen sexo con mujeres pudieron haberlo tenido con hombres en el pasado o tenerlos en el futuro (recordar que la se-xualidad es dinámica). Por ejemplo, en el $20 \%$ de las pacientes que refieren no haber tenido relaciones sexuales con varones se ha documentado ADN de HPV y lesions escamosas intraepiteliales $(\mathrm{SIL})^{20}$.

Como mencionamos previamente y probablemente por las barreras descriptas, esta población no suele alcanzar los estándares de rastreo de ésta entidad clínica, por lo que recomendamos hacer hincapié en que a toda paciente e independientemente de su orientación sexual, se le debe recomendar realizar rastreo de cáncer de cérvix. 
Rastreo de cáncer anal en la población que practica el coito anal receptivo

Respecto del cáncer anal, vale destacar que tiene alta asociación con el número de parejas sexuales a lo largo de la vida y con la infección por los virus HPV y de la inmunodeficiencia humana $(\mathrm{VIH})^{21}$. Ver tabla 5. La incidencia anual de cáncer anal en hombres que tienen sexo con hombres se estima en 35/100.000 (y el doble en los infectados por VIH) similar al del carcinoma de cérvix antes de la instauración de programas masivos de detección a través de la técnica de Papanicolau, luego de lo cual ésta se redujo a su quinta parte ${ }^{22}$.

Tabla 5: factores de riesgo de cáncer anal.

\begin{tabular}{l|c|c|c}
\multicolumn{2}{c|}{ Antecedente } & Mujeres & Varones \\
\multirow{2}{*}{$\begin{array}{l}\text { Número de parejas sexuales } \\
\text { a lo largo de la vida }\end{array}$} & $\mathbf{4}$ a $\mathbf{9}$ & $2,6(1,7$ a 4) & NS \\
\cline { 2 - 4 } & Más de $\mathbf{1 0}$ & $4,5(2,7$ a 7,4$)$ & $2,5(1,1$ a 5,5$)$ \\
\hline Primera relación anal antes de los $\mathbf{3 0}$ & $3,4(1,7$ a 6,6$)$ & NS \\
\hline Condilomas anales & $11,7(4,7$ a 28,9$)$ & $8(1,8$ a 35,9) \\
\hline Infección por VIH & $1,7(1,1$ a 2,8$)$ & $3,1(1,6$ a 6,1$)$ \\
\hline
\end{tabular}

VIH: virus de la inmunodeficiencia adquirida. Fuente: Frischy $\mathrm{M}$ col. Sexually transmitted infection as cause of anal cancer. N Engl J Med 1997;337;1350-8.

En la mucosa anal de los pacientes que practican sexo anal receptivo es común la presencia de HPV y de cambios displásicos; lo que ocurre en el $92 \%$ de los VIH-positivos y en el $66 \%$ de los negativos. EL VIH exacerba los efectos del HPV y se asocia a lesiones escamosas intraepiteliales de mayor grado (SIL); mientras que el paso de lesiones de bajo a alto grado se acelera en presencia de $\mathrm{VIH}^{13}$. Vale destacar que la anoscopía y la citología anal (PAP anal) especialmente en pacientes $\mathrm{VIH}$ positivos o en quienes tienen lesiones (ej. condilomas) anales tienen una costo-efectividad similar a otras prácticas preventivas semejantes $^{13,24}$. La técnica de recolección es sencilla ${ }^{15}$, no requiere preparacion previa salvo la abstención de relaciones sexuales anales receptivas y puede ser tomada con el paciente en decúbito lateral con las rodillas contra su pecho. Un hisopo de Dacron (NR) debe ser introducido en el canal anal a 5 a $6 \mathrm{~cm}$ de profundidad. Luego, a medida que se lo va presionando contra las paredes de ano y rotando sobre su eje, se lo va extrayendo. Puede ser recogido en forma de citología en medio liquido sobre un vidrio con fijador, de la misma forma que el PAP cervical. El reporte se hace con la clasificación de Bethesda.

Para resumir, la figura 3 esquematiza algunas pautas sugeridas para el control de salud de la población lesbiana, gay y bisexual.

Figura 3: algoritmo sugerido para esquematizar el control de salud en la población lesbiana, gay y bisexual.

\begin{tabular}{|c|}
\hline Rastreo de trastornos alimentarios, \\
anímicos, ansiedad y abuso de sustancias \\
Historia Sexual \\
TODAS LAS PRACTICAS PREVENTIVAS \\
DE LA POBLACION GENERAL \\
\hline Si corresponde: \\
Rastreo VIH/ETS \\
Inmunización contra Hepatitis A y B \\
Pap anal ante historia de sexo anal receptivo \\
e infección por VIH y/0 condiciones
\end{tabular}

\begin{tabular}{|c|c|}
\hline BAJO RIESG0 \\
Revaluación anual, \\
PAP cada 203 años
\end{tabular}$\quad \begin{gathered}\text { ALTO RIESGO } \\
\text { Revaluación semestral, } \\
\text { serología y PAP anual }\end{gathered}$

Elaboración propia sobre la base de: Knight D. Health Care Screening for Men Who Have Sex with MenAm Fam Physician 2004;69:2149-56.

"'Por ejemplo, datos de Censo 2000 informan que en EE.UU. estaban criando niños el $34,3 \%$ de las parejas de lesbianas, el $22,3 \%$ de las parejas de gays y el $44 \%$ de las de heterosexuales; destacándose que el $41 \%$ de las parejas homosexuales que estaban criando niños estaban juntas hacía más de cinco años.

Si bien siempre ${ }^{\star \star \star}$ ha habido niños criados en familias homoparentales ${ }^{25}$, en los últimos años y el contexto del debate por la igualdad de derechos de las minorías sexuales y su traducción en algunas legislaciones, ha tomado fuerza el debate sobre el desarrollo y la salud de estos niños. En este sentido, vale destacar los resultados de dos trabajos observacionales:

El de Wainright y col. ${ }^{26}$ evaluó una muestra de 12000 adolescentes de dos escuelas de 80 municipios de EE.UU. seleccionados probabilísticamente a través de una estratificación poli-etápica. Fueron comparados 44 adolescentes criados por dos mujeres viviendo en pareja "tipo matrimonial" con 44 controles. Ambos grupos tuvieron similares resultados escolares, puntuaciones en cuestionarios de autoestima, ansiedad y depresión; así como de otras variables menos objetivas como la valoración de relaciones familiares positivas, calidez parental, la percepción de sentirse cuidados, autonomía e integración vecinal; y mayor conexión escolar.

El de Golombok y col. ${ }^{27}$, que en el contexto de la evaluación de 14000 madres inglesas comparó niños de cinco a siete años de edad criados en tres tipos de familia (39 con madre lesbiana, 74 con los dos padres heterosexuales y 60 en un hogar monoparental con madre heterosexual). Fueron medidos varios indicadores, entre ellos el reporte de conductas anormales por el maestro y la prevalencia de trastornos psiquiátricos; y otros más "blandos" como involucramiento emocional de la madre, frecuencia de conflictos, supervisión del niño y autoestima de niño. Las únicas diferencias identificadas fueron atribuibles a haber sido criados en un hogar monoparental y no homoparental.

En este sentido, y en consonancia con la postura de la Sociedad Argentina de Pediatría en un documento divulgado durante $2010^{28}$ en el contexto del debate por la Ley de "Matrimonio entre personas de mismo sexo" podríamos reasegurar a los pacientes que no existe evidencia de que los niños criados por uno o más progenitores homosexuales tengan un desarrollo emocional, cognitivo, social o sexual diferente a los niños criados por padres heterosexuales (recomendación tipo $B$ derivada de estudios observacionales).

\section{Conclusión}

El profesional de la salud que atiende una persona con OSM puede sentirse incómodo al atenderla y/o puede sentir que no está capacitado para responder a alguna de sus inquietudes y/o para dar algún consejo de salud.

Es importante que frente a esta eventual situación pueda admitir sus limitaciones, comunicarlas a su paciente y eventualmente decirle que va a investigar el tema o discutir con otros colegas.

No debería alarmarse por que ocurra esto ya que seguramente el paciente va a sentir que nos estamos preocupando por él y probablemente sea una de sus mejores experiencias con el sistema de salud.

${ }^{\star}$ Ver glosario 


\section{Referencias:}

1. Klein F y col. Sexual orientation: A multi-variable dynamic process. J. Homosexuality. 1985; 11: 35-49. Disponible en URL:

http://www.gaydata.org/04_Measures/ms006_Klein/ms006_Klein.html

2. Kinsey A y col. (1948/1998) Sexual Behavior in the Human Male; (1953/1998) Sexual Behavior in the Human Female; y Gebhard P y col. (1979/1998) The Kinsey Data:

Marginal Tabulations of 1938-1963 Interviews Conducted by the Institute for Sex Research. Philadelphia: W.B. Saunders; Bloomington, IN: Indiana U. Press. Versión resumida disponible en URL: http://www.iub.edu/ kinsey/resources/ak-data.html\#Findings

3. Laumann O y col. The Social Organization of Sexuality: Sexual Practices in the United States. Chicago: University of Chicago Press, 1994. Citado por: Dean L, et al.

Lesbian, Gay, Bisexual, and Transgender Health: Findings and Concerns. Journal of the Gay and Lesbian Medical Association, Vol. 4, No. 3, 2000.

4. Knight D. Health Care Screening for Men Who Have Sex with MenAm Fam Physician 2004;69:2149-56.

5. American Psychiatric Association. Diagnostic and statistical manual of mental disorders. 2. Washington,

DC: Author; 1973

6. Pearling L The stress process revisited: reflections on concepts and their interrelationships. In: Aneshensel C y col. Handbook of sociology of mental health. New Yorl. Kluwer Academic/Plenum; 1999b p. 309-415. Citado por: Meyer I. Prejudice, Social Stress, and Mental Health in Lesbian, Gay, and Bisexual Populations: Conceptual Issues and Research Evidence. Psychol Bull. 2003 September ; 129(5): 674-697.

7. Bazán O. Historia de la homosexualidad en la Argentina. De la Conquista de América al siglo XXI.2005, Marea Editorial.

8. Diagnostic and Statistical Manual of Mental Disorders DSM-IV-TR Fourth Edition (Text Revision). Washington DC. American Psychiatric Association, 2000.

9. King M y col. A systematic review of mental disorder, suicide, and deliberate self harm in lesbian, gay and bisexual people. BMC Psychiatry. doi:10.1186/1471-244X-8-70. Disponible en URL: http://www.biomedcentral.com/1471-244X/8/70 (último acceso 20/10/10).

10. Tjepkema M. Health care use among gay, lesbian and bisexual Canadians. Component of Statistics Canada Catalogue no. 82-003-X Health Reports. March 19 , 2008. Disponible en URL:

http://www.google.com.ar/url?sa=t\&source=web\&cd=1\&ved=0CBUQFjAA\&url=http\%3A\%2F\%2Fwww.glhv.org.au\%2Ffiles\%2Fcanada_glb_health_use.pdf\&rct=j\&q=canadian\%202003\%202006\%20lesbian\%20pap\%20regular\%20doctor\&ei=hm7QTM-QC4H48Aa0q9ncBg\&usg=AFQjCNHvj-EibpzPuBHLKiwW7gkvdliVYA\&cad=rja (último acceso: $02 / 11 / 10)$.

11. Gay and Lesbian Medical Association. Guidelines for care of lesbian, gay, bisexual and transgender patients.

http://ce54.citysoft.com/_data/n_0001/resources/live/GLMA\%20guidelines\%202006\%20FINAL.pdf

12. Mravcak S. Primary care for lesbians and bisexual women. Am Fam Physician. 2006; 74:279-86.

13. Social and sexual comitee members. Lesbian Health Guidelines. Journal Soc Obstet Gynaecol Can 2000;22(3):202-5.

14. Ambrose M and Ladewski L. A University of Michigan Resource Guide Published by BGLAM. Caring for Lesbian, Gay, Bisexual, and Transgender Patients. 2005.

Disponible en URL http://www.umich.edu/ bglam/Resource-Guide-PRINT.pdf (último acceso 30/12/10).

15. Health Care Needs of Gay, Lesbian, Bisexual, \& Transgender Patients: En South Paul J, et.al. Current Diagnosis and Treatment in Family Medicine. Mc Graw Hill. www.accessmedicine.com (ultimo acceso 12/10/08).

16. Dean L, et al. Lesbian, Gay, Bisexual, and Transgender Health: Findings and Concerns. Journal of the Gay and Lesbian Medical Association, Vol. 4, No. 3, 2000. http://ce54.citysoft.com/_data/n_0001/resources/live/Columbia-GLMA\%20White\%20Paper.pdf

17. Taking a sexual history. Department of Health and Human Services, Centers for Disease Control and Prevention. Accessed November 17, 2005, at: http://www.cdc.gov/std/see/HealthCareProviders /SexualHistory-H.pdf.

18. Sexually transmitted diseases treatment guidelines 2002. Centers for Disease Control and Prevention. MMWR Recomm Rep 2002; 51(RR-6):1-78.

19. Bailey J. Lesbians and Cervical Screening. British Journal of General Practice., June 2000.

20. Health Care Needs of Gay, Lesbian, Bisexual, \& Transgender Patients: En South Paul J, et.al. Current Diagnosis and Treatment in Family Medicine. Mc Graw Hill. www.accessmedicine.com

21. Frischy M col. Sexually transmitted infection as cause of anal cancer. N Engl J Med 1997;337;1350-8.

22. Darragh T y col. The ABCs of anal-rectal cytology. PAP/NGC Program Review. College of American Pathologists. Disponible en URL: http://www.cap.org

23. Goldie $S$ y col. The Clinical Effectiveness and Cost-effectiveness of Screening for Anal Squamous Intraepithelial Lesions in Homosexual and Bisexual HIV-Positive Men. JAMA. 1999;281:1822-1829.

24. Goldie $S$ y col. Cost-effectiveness of screening for anal squamous intraepithelial lesions and anal cancer in human immunodeficiency virus-negative homosexual and bisexual men. Am J Med. 2000 Jun 1;108(8):634-41.

25. Pawelski $\mathrm{J}$ y col. The effects of marriage, civil union, and domestic partnership laws o the health and wellbeing of children. Pediatrics Vol. 118, July 2006 .

26. Wainright $\mathrm{J}$ y col. Psychosocial adjustment, school outcomes, and romantic relationships of adolescents with same-sex parents. Child Dev. 2004;75:1886-1898.

27. Golombok Set al. Children with lesbian parents: a community study. Dev Psychol. 2003;39:20-33.

28. Sociedad Argentina de Pediatría, Grupo de Trabajo Derechos del Niño, Comité Nacional de Familia y Salud Mental, Comité Nacional de Pediatría Ambulatoria. Aporte de

la Sociedad Argentina de Pediatría a los Legisladores. 2010. Disponible en URL: http://www.sap.org.ar/docs/mmsexo.pdf (último acceso 11711/10). 